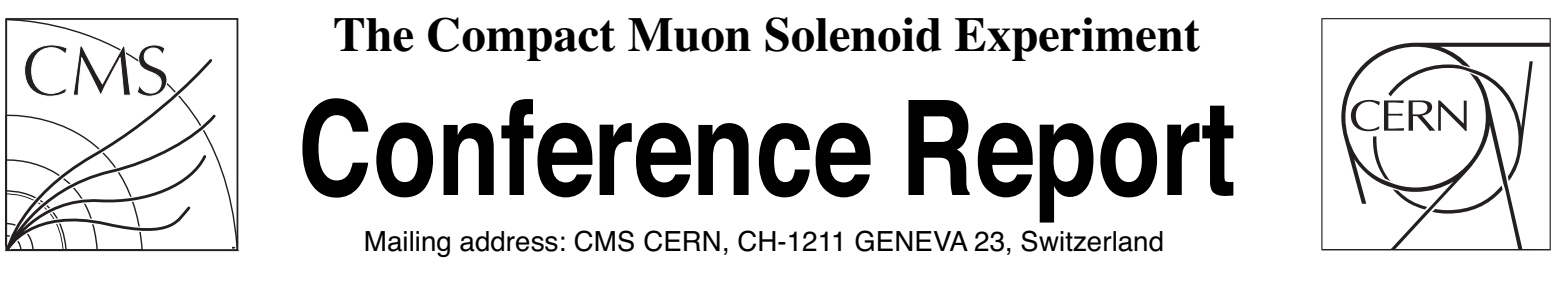

11 April 2020 (v5, 19 December 2020)

\title{
Comparative aging studies on a Single Wire Proportional Chamber
}

\author{
Davide Fiorina for the CMS Collaboration
}

\begin{abstract}
In order to extend previous studies and test additional variables playing a role in the aging processes, a single wire proportional chamber was specially designed. In this contribution, the author will present the result of two types of tests performed on this single wire proportional chamber. During the first test, the single wire proportional chamber was irradiated with X-rays and alpha particles with the same hit rate until the same collected charge ([per-mode=symbol]1.4) was reached. Nevertheless the performance loss during the x-ray irradiation was larger, traces of polymers were found on the wire only in the case of alpha irradiation. The second test was performed irradiating the single wire proportional chamber with alphas and X-rays, but this time with the same anodic current i.e. very different hit rate. The irradiation continued until the wire chamber lost about half of the initial gas gain. In the case of alpha irradiation the integrated charge needed for the goal was lower with respect to the one needed during the $\mathrm{x}$-ray radiation: [per-mode=symbol] 9.5 and [per-mode=symbol] 33.0 , respectively. However, the electron microscope analysis revealed, once again, a large polymer deposit on the alpha irradiated wire while no deposit was found on the X-ray irradiated one.
\end{abstract}

Presented at INSTR20 Instrumentation for Colliding Beam Physics 


\section{Comparative aging studies on a single wire proportional chamber}

To cite this article: D. Fiorina 2020 JINST 15 C07008

View the article online for updates and enhancements.

\section{IOP ebooks}

Bringing together innovative digital publishing with leading authors from the global scientific community. Start exploring the collection-download the first chapter of every title for free.

This content was downloaded from IP address 193.204 .40 .97 on 19/12/2020 at 15:04 


\section{Comparative aging studies on a single wire proportional chamber}

\section{Fiorina on behalf of CMS collaboration}

Università degli studi di Pavia,

via A. Bassi 6, Pavia, Italy

INFN sezione di Pavia,

via A. Bassi 6, Pavia, Italy

E-mail: davide.fiorina@cern.ch

Aвstract: In order to extend previous studies and test additional variables playing a role in the aging processes, a single wire proportional chamber was specially designed. In this contribution, the author will present the result of two types of tests performed on this single wire proportional chamber. During the first test, the single wire proportional chamber was irradiated with X-rays and alpha particles with the same hit rate until the same collected charge $(1.4 \mathrm{mC} / \mathrm{cm})$ was reached. Nevertheless the performance loss during the x-ray irradiation was larger, traces of polymers were found on the wire only in the case of alpha irradiation. The second test was performed irradiating the single wire proportional chamber with alphas and X-rays, but this time with the same anodic current i.e. very different hit rate. The irradiation continued until the wire chamber lost about half of the initial gas gain. In the case of alpha irradiation the integrated charge needed for the goal was lower with respect to the one needed during the x-ray radiation: 9.5 and $33.0 \mathrm{mC} / \mathrm{cm}$, respectively. However, the electron microscope analysis revealed, once again, a large polymer deposit on the alpha irradiated wire while no deposit was found on the X-ray irradiated one.

KeYwORDS: Gaseous detectors; Radiation-hard detectors 


\section{Contents}

1 Introduction 1

2 Aging in gaseous detectors 2

3 Aging tests on single wire proportional chamber 2

4 Results 5

$\begin{array}{llr}5 & \text { Conclusion } & 8\end{array}$

\section{Introduction}

Aging phenomena in gaseous detectors were intensely studied in the last century for improving the reliability and the long term operation of the technologies in order to use them to obtain relevant physics results for various experiments. The last large review of aging in gaseous detectors was held in 2001 [1], when the scientific community was entering the LHC era (started in 2010) where the detectors had to face an unprecedented harsh radiation environment.

Nowadays with the refined procedures for testing materials and assembly detectors, aging issues seem to be under control and many techniques for preventing or correcting them are well known [2]. However, we are far away from the complete microscopic and macroscopic understanding of the phenomenon [3,4]. Nevertheless, many new aging tests were carried out in order to validate different gaseous detector technologies and designs in the LHC era (some examples can be found in [5-9]), to the author's knowledge, not the same effort was spent on the basic understanding of the aging phenomena in the last two decades. On the other hand, in the near and far futures, we shall face new challenges due to the increase of the luminosity and energy of colliders. For example, HL-LHC will deliver a radiation dose to the CMS detector 10 times higher than today's LHC [10]. For possible future colliders, such as High-Energy LHC and Future Circular Collider the foreseen dose will be respectively 40 and 200 times more than at today's LHC [11].

This work aims to be the first of a long series and stimulate the R\&D studies of such a delicate issue. A set of aging tests was performed on a single wire proportional chamber in order to investigate some macroscopic aging variables such as the irradiating particle ionization power, the anodic current density, and the hit rate. These tests were carried out with an original way to accelerate the aging in a gaseous detector. This work is part of a larger set of aging tests performed on different gaseous detector technologies, a part of it was already presented at a conference [12] and more results shall be presented in the future. 


\section{Aging in gaseous detectors}

In general, we call aging any type of degradation in the detector performance after an irradiation period. In particular, the "classical aging effects" are due to the chemical processes occurring in the avalanche plasma during the electron multiplication, this leads to the formation of deposits on the electrodes $[3,13]$. During the avalanche process, the electrons multiply by ionization of atoms and molecules, de-excitation and photon absorption processes. Since the mean ionization energy is of the order of tens $\mathrm{eV}$ while the mean molecular dissociation energy is of the order of $\mathrm{eV}$, during the avalanche there will be the presence of many molecular fragments surrounding the electron/ion pairs. In some cases, these fragments are free radicals, i.e. atomic and molecular species with one or more unsatisfied covalent bonds. They could recombine into polymers which can be conductive or insulating and they could deposit on the sensitive surfaces of the detector, such as the electrodes, and then worsen the detector performance.

It is therefore possible to understand that the choice of the gas mixture is of fundamental matter for the long term operation of the detector. Nowadays, the cleanest gas mixture from the aging point of view is $\operatorname{Ar}(\mathrm{Xe}) / \mathrm{CO}_{2}$, indeed there is no well-established mechanism that could lead to the formation of deposit $[4,13,14]$. Although the gas mixture can be controlled, it is more difficult to contain the pollution of the gas system. Indeed many materials present in the gas systems can be susceptible to outgassing, i.e. release of some molecules inside the gas volume. Silicon and hydrocarbon-based molecules revealed to be very dangerous also in aging-free gas mixtures. Silicon, in particular, revealed to be very dangerous both because it is used in the production procedure of many gas system components and because small quantities can be fatal for the detector. Moreover, in some cases, the source of the Si-based pollutant has not been clearly identified [4].

The collected charge, $\mathrm{mC} / \mathrm{cm}$ for single and $\mathrm{mC} / \mathrm{cm}^{2}$ for double dimensional anodes, is usually used to quantify the amount of irradiation in gaseous detectors and it is convenient to compare different aging effects. In particular, it is possible to roughly correlate the 2-D collected charge with the 1-D one by multiplying the latter by the length of the wire orthogonal dimension of the irradiated area.

\section{Aging tests on single wire proportional chamber}

The tests presented in this work aim to extend the knowledge on the aging issue by studying some of the many macroscopic variables which take part in these processes. The tests intended to understand the different aging properties of X-rays $\left(5.9 \mathrm{keV}\right.$ photons from a ${ }^{55} \mathrm{Fe}$ source which have a low ionization power) and alpha particles (5.5 MeV alphas from a ${ }^{241} \mathrm{Am}$ source which have a high ionization power). In order to discriminate the effect beyond the pure collected charge, the comparison has been made under two different irradiating conditions: at the same hit rate with the detector collecting the same amount of charge, and at the same anodic current with the detector losing the same amount of effective gas gain due to aging. All the four tests were performed with an original way to accelerate the aging in laboratory, indeed two types of glues were inserted in a stainless steel cylinder where the gas $\left(\mathrm{Ar} / \mathrm{CO}_{2} 70 \% / 30 \%\right)$ is flushed before entering the detector. The glues were dried for at least 24 hours before being flushed and they are heated up to around $50^{\circ} \mathrm{C}$ to stimulate the releasing of pollutants inside the gas volume. These are hydrocarbon (figure 1) 
and Si-based (figure 2) molecules, used in order to cover a relatively wide range of contaminants that it is possible to find in many potentially dangerous materials. These tests were performed with<smiles>C=C(C)C(=O)OC</smiles>

Figure 1. MethylMethacrylate molecule from 3140 RTV Coating ${ }^{\circledR}$.

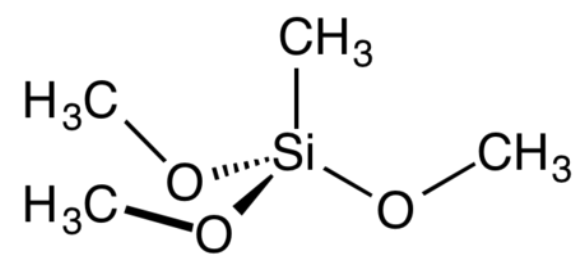

Figure 2. Methyltrimethoxysilane molecule from Acrifix 1R $0192^{\circledR}$.

the same single wire proportional chamber specifically designed for the purpose; after each test the detector wire is changed and all the pieces are washed in an ultrasonic bath with isopropyl alcohol in order to remove any trace of silicon pollutants and most of the hydrocarbons [4, 9]. The used wire is sampled and analysed using an electron microscope in order to find traces of deposit and an energy dispersive $\mathrm{x}$-ray analysis is performed to measure the concentration of the atomic specimen in these deposits [15].

A single wire proportional chamber was specifically designed in order to perform these tests, a detector design ensuring easy operation and a fast mounting and disassembly was required for these tests. The first effort to produce such a detector [16] was abandoned due to the instability of the gas gain. The new design, shown in figure 3, consists in a $26 \mathrm{~cm}$ long steel cylinder of $2.8 \mathrm{~cm}$ diameter. $4 \mathrm{~mm}$ diameter holes are made on one side of the detector to let alpha particles inside the gas volume, the gas tightness is ensured by a thin polyamide foil. This chamber provided the operation stability for a long term and a relative easiness in mounting: after some trials, the complete disassembly, sampling, washing, and mounting of the chamber took just one hour.

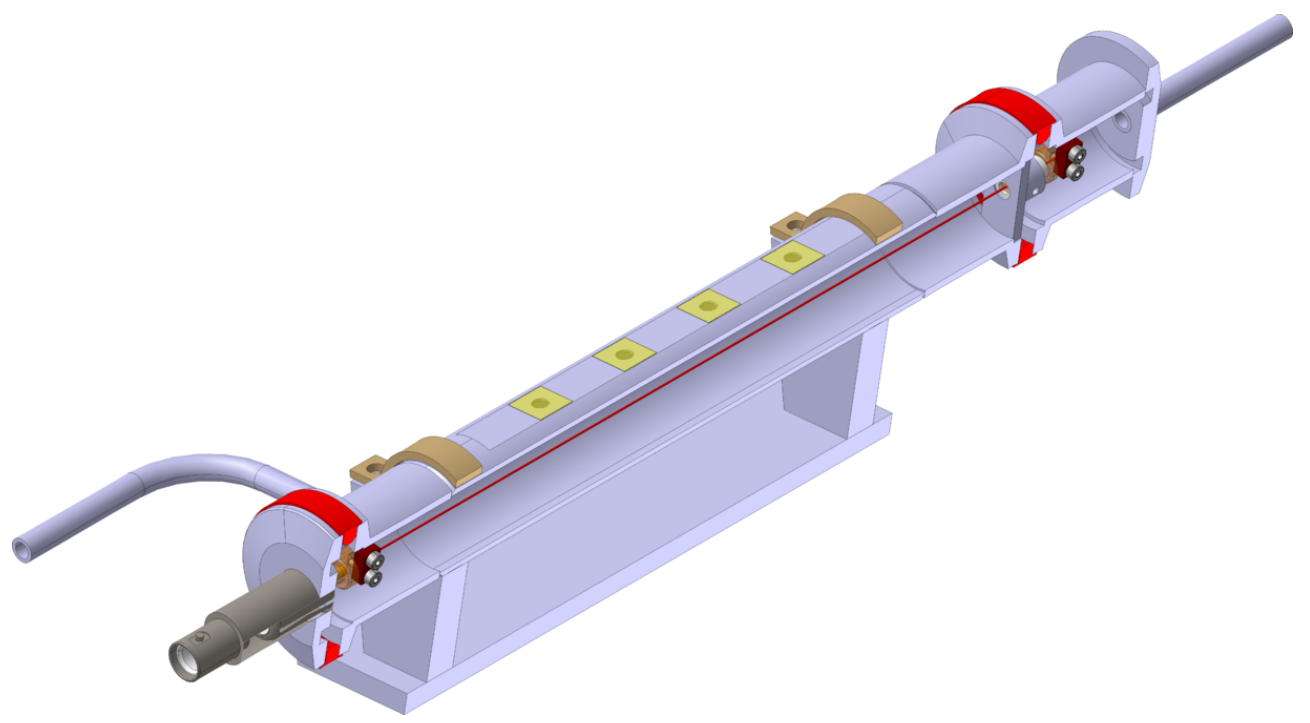

Figure 3. Section of the single wire proportional chamber. 
To monitor the detector performance, charge spectra are acquired for both X-rays and alphas irradiations, each acquired spectrum contains data for 30 minutes irradiation and it is fitted with three Gaussian functions. In the x-ray irradiation, two Gaussian functions are used to fit the photopeak (at $5.9 \mathrm{keV}$ ) and the Argon escape peak (at around $3 \mathrm{keV}$ ), for the alpha charge spectra; two Gaussian functions were used for the alpha peak because of the many ${ }^{241} \mathrm{Am}$ alpha emission lines $(5.5 \mathrm{MeV})$ and the wire chamber energy resolution, the resulting signal peak is well fitted with two Gaussian functions. The third Gaussian, present in both the cases, refers to the pedestal peak. The distance of the signal peak from the pedestal is proportional to the effective gas gain of the detector and it is monitored during the data taking period in order to quantify the aging occurring in the gas volume. Furthermore, since the detector gain depends on the gas temperature and pressure, an Arduino based climate monitor is used to measure these parameters and to correct the gain using a power function [5, 16]. Figure 4 shows the scheme of the experimental setup, while figure 5 shows an example of the charge spectrum in the case of the ${ }^{55} \mathrm{Fe}$ irradiation.

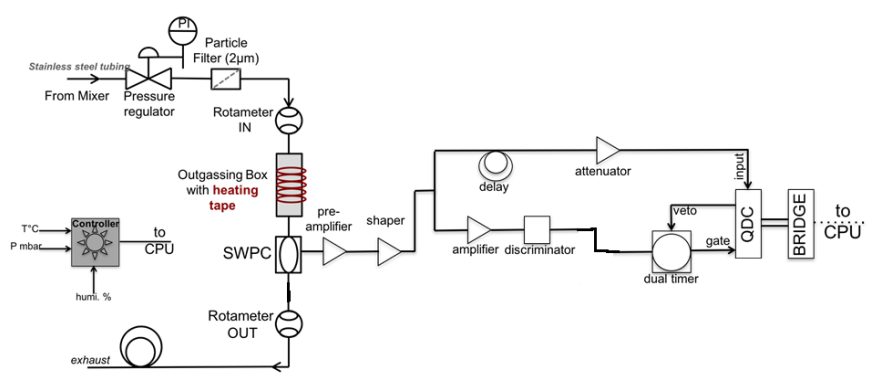

Figure 4. Schematic view of the experimental setup.

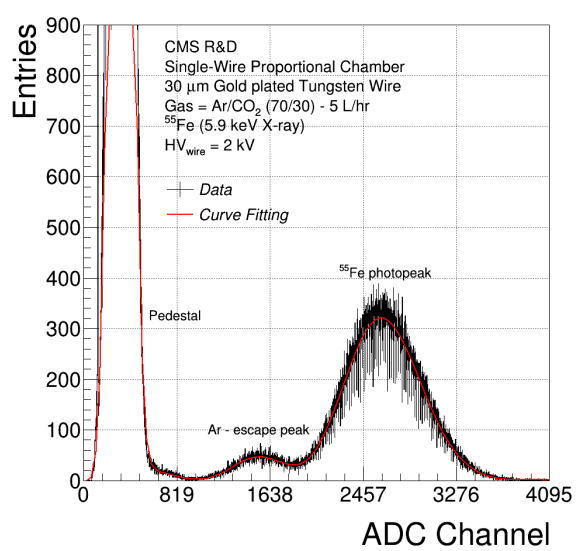

Figure 5. Example of a charge spectrum of the detector irradiated with a ${ }^{55} \mathrm{Fe}$ source.

Table 1 briefly summarizes some of the interesting aging parameters of the four tests presented in this work.

Table 1. Resume of some operating parameters of the tests.

\begin{tabular}{|c|ccc|c|c|}
\hline \multirow{2}{*}{} & \multicolumn{2}{|c|}{ Same hit rate test } & \multicolumn{2}{c|}{ Same anodic current test } \\
\cline { 2 - 5 } & X-rays & Alpha & X-rays & Alpha \\
\hline Operating voltage $(\mathrm{V})$ & 1950 & 1950 & 1900 & 1900 \\
\cline { 1 - 1 } Effective gas gain $\left(\times 10^{4}\right)$ & 3.2 & 3.6 & 2.7 & 2.4 \\
\cline { 1 - 1 } Hit rate $(\mathrm{kHz})$ & 0.65 & 0.61 & 10.50 & 0.62 \\
\cline { 1 - 1 } Current density $(\mathrm{nA} / \mathrm{cm})$ & 6.4 & 130.8 & 83.8 & 87.0 \\
\cline { 1 - 1 } Integrated charge $(\mathrm{mC} / \mathrm{cm})$ & 1.4 & 1.4 & 9.5 & 33.0 \\
\hline
\end{tabular}




\section{Results}

Figures 6 (a) and (b) show the plots of the effective gas gain evolution during the irradiation period. When irradiating with X-rays, the detector lost around $20 \%$ of the nominal gain while, when irradiating with alphas, the gain drop is about $10 \%$, both the tests accumulated the same amount of charge $(1.4 \mathrm{mC} / \mathrm{cm})$ but the first one lasted about 20 times longer due to the very different current density on the anode (see table 1). Figures 6 (c) and (d) show the plots of the tests performed at the same anodic current. During both the irradiation periods the detector lost (more or less) the same amount of performance: $45 \%$ drop in the effective gas gain. It is possible to notice in the x-ray plot that, around $21 \mathrm{mC} / \mathrm{cm}$, the gain stopped to drop. This effect is due to an unexpected power loss that lasted for a whole weekend. The high voltage was off for about 2 days while the gas continued flushing, so the hypothesis is that the gas flow removed some of the upper layers of the polymer deposit causing a slower growth of it during the next days. Taking note of this, it may say that the $\mathrm{X}$-rays irradiation took between 2 and 3 times the accumulated charge in order to reach the same gain loss. Furthermore, it may be noted that, at the same accumulated charge $(9.5 \mathrm{mC} / \mathrm{cm})$ the detector irradiated with photons lost just $25 \%$ of the gas gain vs. the $45 \%$ of the alpha irradiation. After the irradiation, the used wire is sampled and analysed with a scanning electron microscope, an electron dispersive $\mathrm{x}$-ray analysis is also performed in order to measure the relative concentration of atomic specimens in interesting areas. Figure 7 shows two pictures of an unused wire as a reference, the wire is relatively smooth and the imperfections are due to the gold plating process of the tungsten, indeed only gold is detected on the wire. Figures 8 (a) and (b) show the pictures from the tests with the same hit rate, it may be noticed that the x-ray irradiated wire presents no visible deposit, in fact, no other atomic specimens were found but gold. On the other hand, the alpha irradiated wire present a wide silicon deposit (no carbon detected) on the whole irradiated area, the layer thickness varies from $1.5 \mu \mathrm{m}$ on the wire portion directly exposed to the incoming particles to $0.7 \mu \mathrm{m}$ on the opposite side. Finally, figures 8 (c) and (d) show wire pictures from the tests performed at the same anodic current. Also in this case, on the x-ray irradiated wire, no deposit was found, while in the alpha irradiated one, a very thick (several $\mu \mathrm{m}$ ) silicon deposit was seen.

By analysing the gain as a function of the collected charge, some conclusions may be drawn. During the same hit rate test, the gain drop in the X-rays irradiation case was more or less the double of the alpha case after the same amount of collected charge. It could be that the number of interactions, in this case, plays a major role with respect to the deposited charge per particle (there is a factor 20 between the two tests in both the variables). Since every interaction is a potential aging trigger, having a lot of them may be more dangerous than having fewer interactions but made by more ionizing particles. However, since the accumulated charge is relatively low for an aging test and also the gain drop is small, the previous hypothesis has to be taken with a grain of salt and has to be confirmed by further tests with more statistics. The result of the same anodic current test was, in a certain way, expected and clearer. The detector aged between 2 and 3 times faster when irradiated by $5.5 \mathrm{MeV}$ with respect to the $5.9 \mathrm{keV}$ photons if the anodic current density is similar. This last result seems to be comparable with the result obtained by the HERA-B muon group [17], where they demonstrated that using a $100 \mathrm{MeV}$ alpha beam, their drift chambers aged twice as fast as when they are irradiated with ${ }^{55} \mathrm{Fe}$. Although the differences between the two tests are many (e.g. gas mixture contained $\mathrm{CH}_{4}$ and $\mathrm{CF}_{4}$, alphas energies are quite different and current densities 


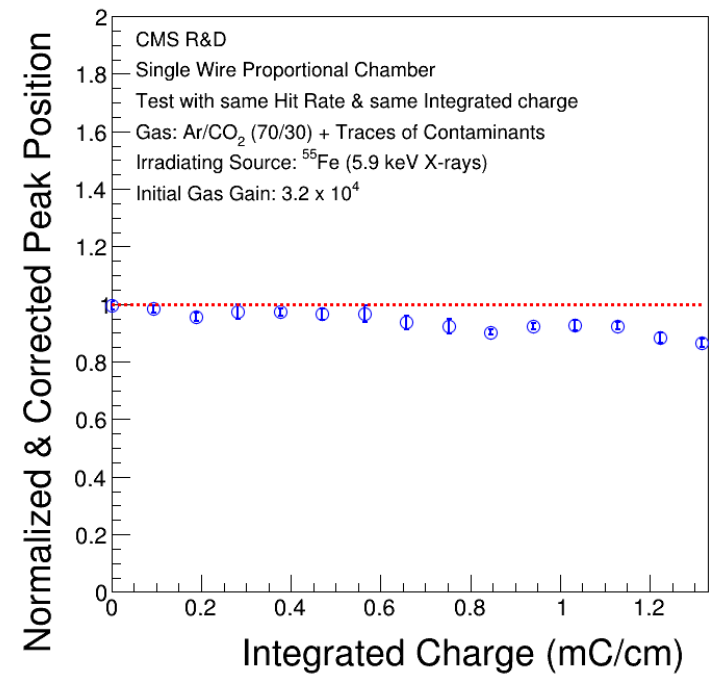

(a)

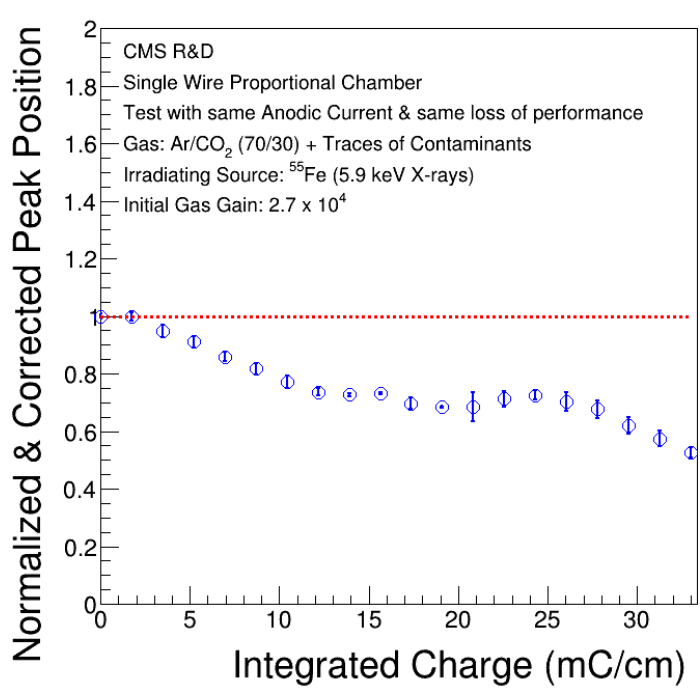

(c)

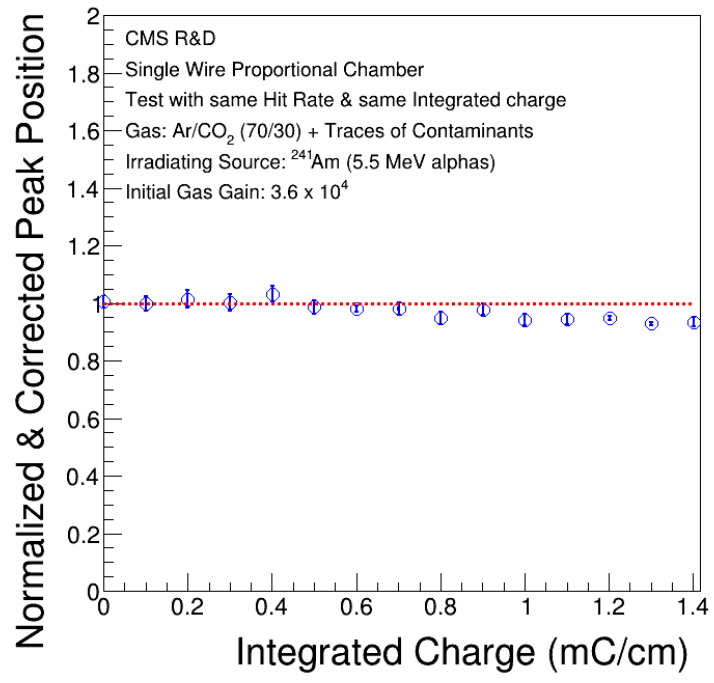

(b)

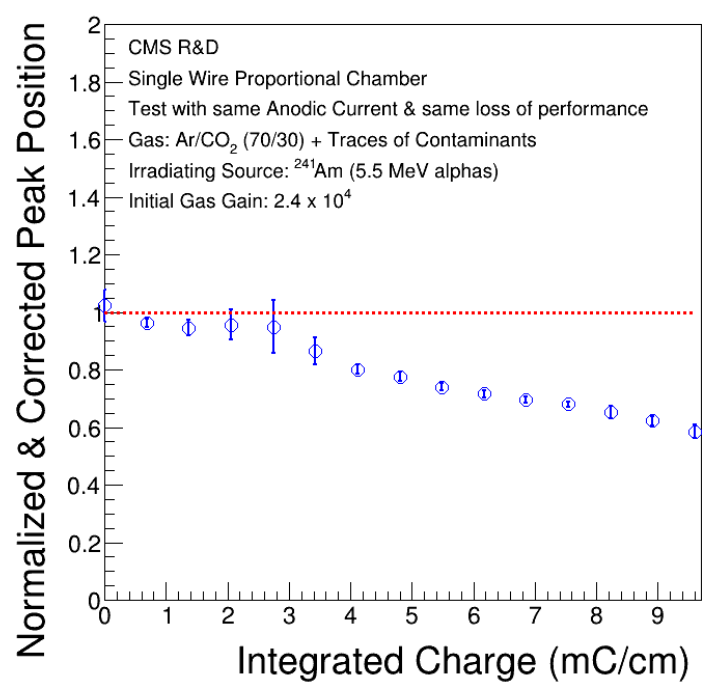

(d)

Figure 6. Effective gas gain evolution as a function of the collected charge. The upper plots refer to the wire chamber test performed with the same hit rate irradiated with X-rays (a) and alpha particles (b). The lower plots refer to the wire chamber test performed with the same anodic current irradiated with X-rays (c) and alpha particles $(\mathrm{d})$.

were the same in the two tests but way higher: $700 \mathrm{nA} / \mathrm{cm}$ ), the result seems to be compatible and attributable to the larger and denser charge released by heavily ionizing particles.

Nevertheless, the electron microscope analysis seems to contradict the previous hypothesis, indeed no traces of contaminants were found in both the X-rays irradiated wires. This seems to be related to the different energy and charge developed during the electron multiplication of photons and alphas, which can completely change the kinematic of the chemical reactions and alter the types 

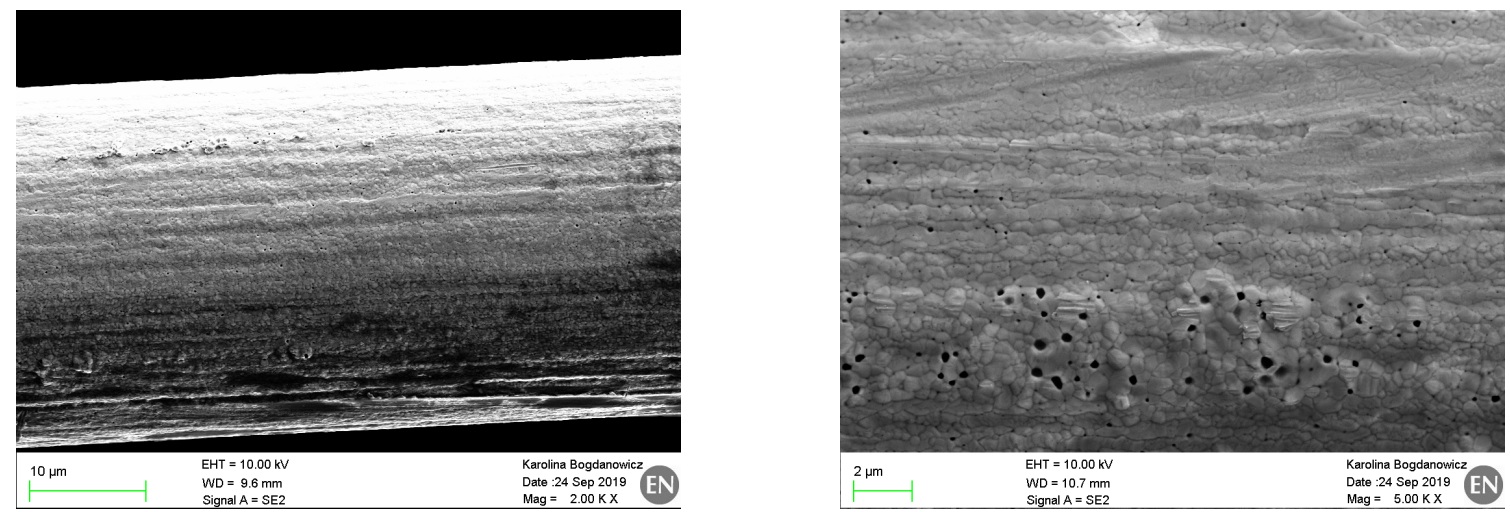

Figure 7. Scanning electron microscope pictures of a new wire portion before the irradiation.
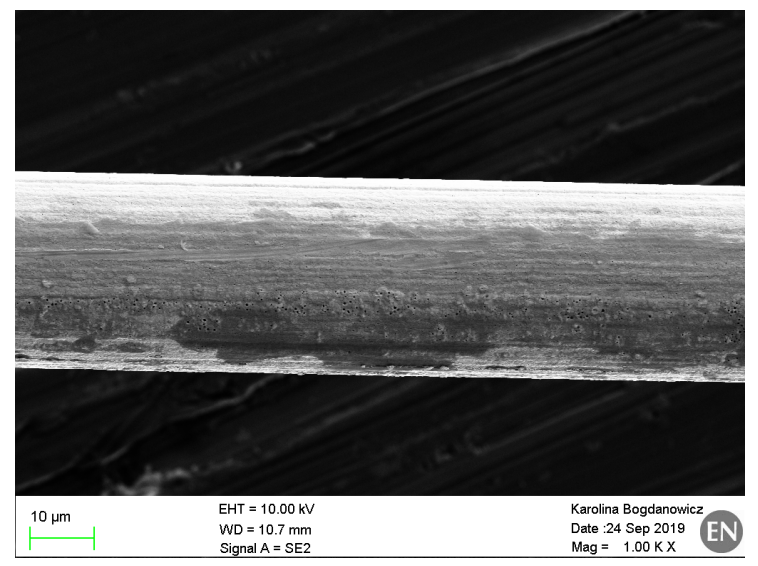

(a)

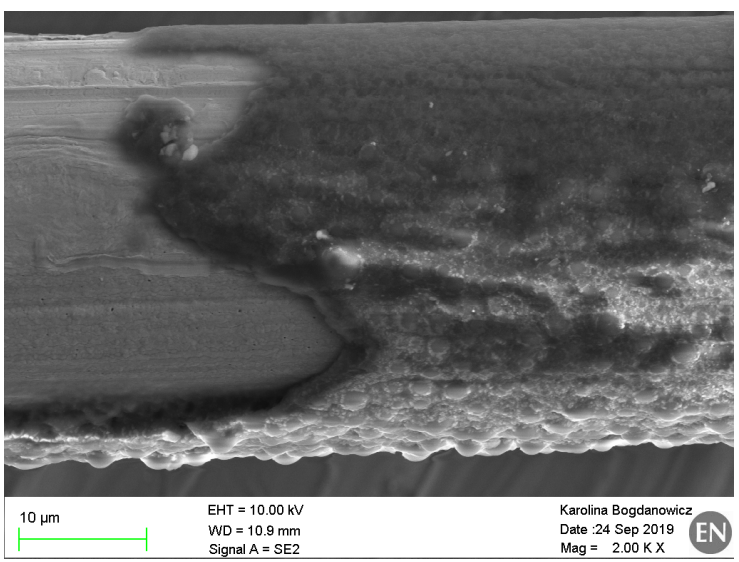

(b)

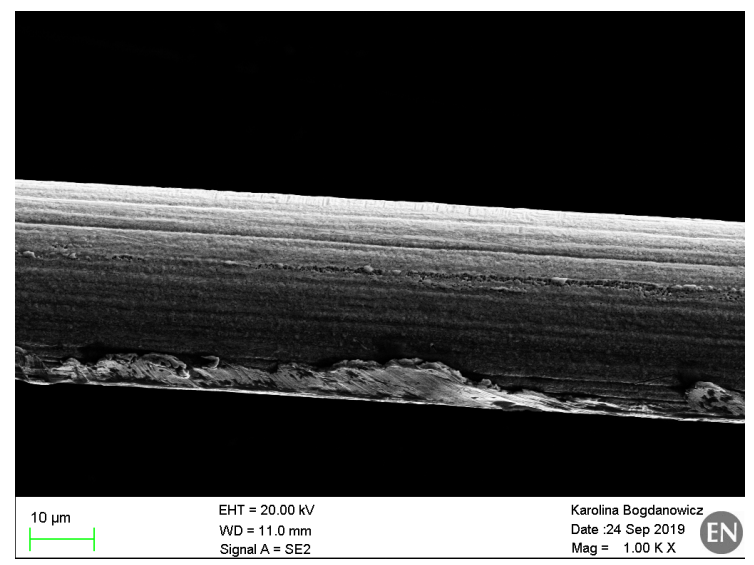

(c)

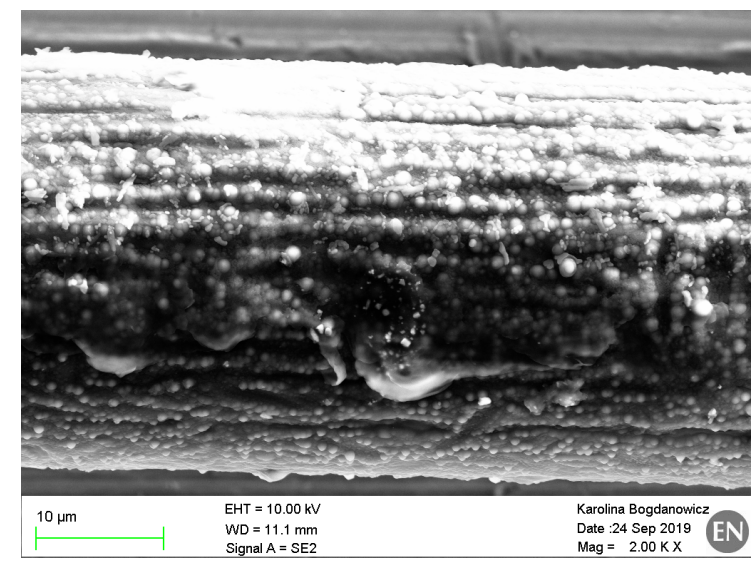

(d)

Figure 8. Scanning electron microscope pictures of the irradiated wires. The upper plots refer to the wire chamber test performed with the same hit rate irradiated with X-rays (a) and alpha particles (b). The lower plots refer to the wire chamber test performed with the same anodic current irradiated with X-rays (c) and alpha particles (d). 
of radicals and polymer created in the avalanche. This can lead, for example, to a lower deposit on the anode with respect to the cathode or it can create polymers on the anode more fragile which can be removed during the delicate sampling operation also if it is made very carefully.

\section{Conclusion}

In this work, two sets of aging tests have been presented on a wire chamber. Using an x-ray and an alpha source, the detectors were irradiated in order to have first similar hit rate, and then similar anodic current. Furthermore, the aging was accelerated by letting some contaminants inside the gas volume.

These early results showed that the absolute number of interactions in the detector could play a major role with respect to the ionizing power in some low statistic cases while leaving space to confirm this hypothesis. Also, the test performed with a similar anodic current revealed a higher aging power of heavily ionizing particles with respect to photons than already observed in [17].

From the electron microscope analysis, it was clear that silicon compounds are way more dangerous than hydrocarbon in a $\mathrm{Ar} / \mathrm{CO}_{2}$ mixture since none of the latter was observed. Also, the lack of polymer deposits in the X-rays irradiated wires could indicate that lightly ionizing particles create polymers in different ways that can deposit on the cathode or can be easily removed also with gentle manipulations.

These tests provide further information about the aging issue in gaseous detectors; nevertheless, since this issue is very complex and the variables are way too many, it is important to continue to expand the knowledge on the issue. In particular, it is important to keep in mind that the next generation of higher energy and higher luminosity experiments will generate stressful environments for the detector technologies like never before.

However, these tests confirmed the better radiation hardness of micro-pattern gaseous detectors with respect to wire chambers also in contaminated gas mixtures [12].

\section{References}

[1] M. Hohlmann, C. Padilla, N. Tesch and M. Titov eds., Proceedings of the International workshop on Aging Phenomena in Gaseous Detectors, Nucl. Instrum. Meth. A 515 (1-2) (2003).

[2] M. Capeans, Aging of gaseous detectors: Assembly materials and procedures, ICFA Instrum. Bull. 24 (2002) 85.

[3] J. Va'vra, Physics and chemistry of aging - early developments, Nucl. Instrum. Meth. A 515 (2003) 1.

[4] M.P. Titov, Radiation damage and long term aging in gas detectors, in Proceedings of the $42^{\text {nd }}$ Workshop on Innovative Detectors for Supercolliders, Erice, Sicily, Italy, 28 September- - 4 October 2003 [physics/0403055].

[5] F. Fallavollita, Triple-Gas Electron Multiplier technology for future upgrades of the CMS experiment: construction and certification of the CMS GE1/1 detector and longevity studies, Ph.D. thesis, CERN-THESIS-2018-349, CERN (2018).

[6] M. Abbrescia et al., Study of long-term performance of CMS RPC under irradiation at the CERN GIF, Nucl. Instrum. Meth. A $\mathbf{5 3 3}$ (2004) 102. 
[7] O. Prokofev et al., Aging tests of full scale CMS muon cathode strip chambers, Nucl. Instrum. Meth. A 515 (2003) 226.

[8] M. Alfonsi et al., Aging measurements on triple-GEM detectors operated with CF-4 based gas mixtures, Nucl. Phys. B Proc. Suppl. 150 (2006) 159.

[9] T. Akesson et al., Aging studies for the ATLAS transition radiation tracker (TRT), Nucl. Instrum. Meth. A 515 (2003) 166.

[10] G. Apollinari et al, High-Luminosity Large Hadron Collider (HL-LHC): Technical Design Report V. 0.1, CERN-2017-007-M.

[11] M. Benedikt and F. Zimmermann, Future Circular Colliders, PoS LeptonPhoton2015 (2015) 052 [CERN-ACC-2015-0165].

[12] F. Fallavollita, D. Fiorina and J.A. Merlin, Advanced Aging study on Triple-GEM Detectors, in Proceedings of the $6^{\text {th }}$ International Conference on Micro Pattern Gaseous Detectors, La Rochelle, France, 5-10 May 2019, J. Phys. Conf. Ser. 1498 (2020) 012038.

[13] J. Va'vra, Review of Wire Chamber Aging, Nucl. Instrum. Meth. A 252 (1986) 547.

[14] J.A. Kadyk, Wire chamber aging, Nucl. Instrum. Meth. A 300 (1991) 436.

[15] https://edms.cern.ch/document/1758512/1

[16] J.A. Merlin, Study of long-term sustained operation of gaseous detectors for the high rate environment in CMS, Ph.D. thesis, CERN-THESIS-2016-041, CERN (2016).

[17] M.P. Titov, M. Danilov, T. Kvaratskhelia, I. Tikhomirov, Y. Zaitsev and Y. Gilitsky, Aging studies for the muon detector of HERA-B, Nucl. Instrum. Meth. A 515 (2003) 202. 